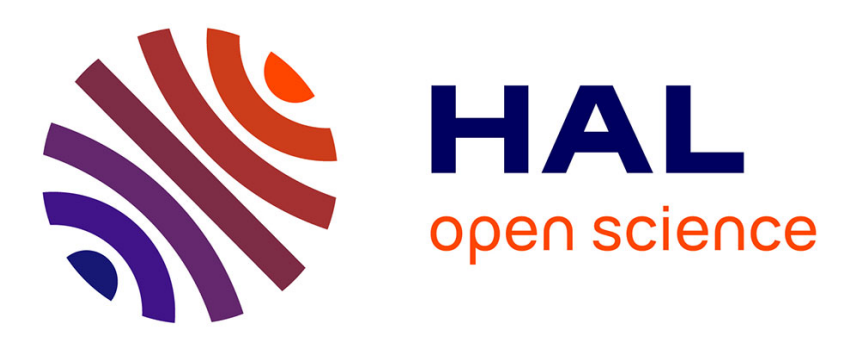

\title{
Approche globale pour l'analyse de la réponse vibratoire d'une transmission par engrenages
}

\author{
Emmanuel Rigaud, Jean Sabot, Joël Perret-Liaudet
}

\section{To cite this version:}

Emmanuel Rigaud, Jean Sabot, Joël Perret-Liaudet. Approche globale pour l'analyse de la réponse vibratoire d'une transmission par engrenages. Revue Européenne des Éléments Finis, 2000, 9 (1-2-3), pp.315-330. hal-00121910

\section{HAL Id: hal-00121910 https://hal.science/hal-00121910}

Submitted on 22 Dec 2006

HAL is a multi-disciplinary open access archive for the deposit and dissemination of scientific research documents, whether they are published or not. The documents may come from teaching and research institutions in France or abroad, or from public or private research centers.
L'archive ouverte pluridisciplinaire HAL, est destinée au dépôt et à la diffusion de documents scientifiques de niveau recherche, publiés ou non, émanant des établissements d'enseignement et de recherche français ou étrangers, des laboratoires publics ou privés. 


\title{
Approche globale pour l'analyse de la réponse vibratoire d'une transmission par engrenages
}

\author{
E mmanuel Rigaud — J ean Sabot — J oël Perret-Liaudet
}

Laboratoire de Tribologie et Dynamique des Systèmes, UMR 5513

Ecole Centrale de Lyon

36 avenue Guy de Collongue, BP 163, 69131 Ecully cedex

Emmanuel.Rigaud@ec-lyon.fr

RÉSUMÉ. Après avoir choisi une transmission type, nous discrétisons l'ensemble de ses composantes par la méthode des éléments finis. Le calcul de sa réponse vibratoire à l'aide d'une méthode spectrale et itérative permet de mettre en évidence les interactions entre ces différentes composantes et d'analyser les mécanismes de transfert entre la surcharge dynamique de denture et la réponse vibratoire du carter. L'excitation des modes de denture et de roulement génère des amplifications de la vitesse quadratique du carter. Ceci nous conduit à proposer des modifications de la transmission (nature des roulements et géométrie $d u$ carter) permettant d'atténuer sensiblement l'état vibratoire du carter dans une large gamme de fréquences.

ABSTRACT. In order to analyse dynamic response of a gearbox, we proposed a method based on the modelling of all the components (gears, shafts, bearings and housing). Forced response of the whole gearbox was computed using a spectral and iterative method. The analysis of coupling mechanisms between dynamic mesh force and mean square vibrational velocity of housing showed that a resonant excitation of modes which have a high potential energy associated with mesh stiffness and bearing stiffnesses leads to the highest vibrational responses. Bearings stiffnesses and mechanical properties of housing have an appreciable influence on these vibrational responses. Our methodology and numerical results allowed to propose new designs which lead to a significant reduction of the mean square vibrational velocity of housing of the gearbox, for a wide range of frequencies.

MOTS-CLÉS: dynamique des engrenages, modélisation, éléments finis, analyse modale, interactions dynamiques, surcharge de denture, vitesse quadratique moyenne du carter.

KEY WORDS : gearbox dynamics, modelling, finite element method, modal analysis, dynamic interactions, mesh force, mean square vibrational velocity of housing. 


\section{Introduction}

Les transmissions par engrenages sont des organes mécaniques couramment utilisés pour transformer et transmettre à un organe récepteur le couple et le mouvement de rotation générés par un moteur. Dans le domaine des transports ou des machines, elles peuvent être à l'origine d'émissions acoustiques importantes que l'on cherche à minimiser.

La réponse vibratoire des transmissions par engrenages est en grande partie générée par une excitation interne liée au processus d'engrènement, l'erreur statique de transmission, dont les sources sont les écarts de géométrie de l'engrenage (défauts de fabrication, erreurs de parallélisme...) et les déformations élastiques des dentures induites par l'application du couple moteur [WEL 79, REM 92]. Ces sources sont à l'origine d'une fluctuation périodique de la raideur d'engrènement, raideur qui résulte du contact entre les roues dentées, et d'une excitation de type déplacement localisée au niveau du contact entre les dents en prise.

En régime de fonctionnement stationnaire, l'erreur statique de transmission génère des surcharges dynamiques sur les dentures qui sont transmises aux lignes d'arbres, aux roulements et au carter de la transmission. Les vibrations induites par l'excitation de ces différentes composantes sont à l'origine de nuisances acoustiques. C'est l'état vibratoire du carter de la transmission qui constitue la principale source du bruit rayonné.

La complexité des mécanismes mis en jeu rend particulièrement difficile le calcul de la réponse vibratoire des carters de transmissions. Bien que la connaissance de cette réponse soit nécessaire à la prédiction du bruit rayonné, les modèles proposés dans la littérature ne prennent en compte que les vibrations de torsion des lignes d'arbres et, plus rarement, les vibrations de flexion des arbres et les déformations des roulements [OZG 88, NER 88, KAR 93]. La réponse du seul engrenage est supposée représentative du comportement acoustique de la transmission complète.

Une autre alternative proposée est de calculer la réponse vibratoire du carter en découplant celle-ci de la réponse des lignes d'arbres [TAK 91, KAT 96]. Le carter nu est alors excité à partir des efforts calculés à l'aide des modélisations précédentes (carter supposé rigide).

Cette approche n'est pas pleinement satisfaisante car les modes propres du carter nu sont fondamentalement différents de ceux du carter équipé des lignes d'arbres. De plus, il convient de vérifier si les propriétés mécaniques du carter n'affectent pas la nature des efforts transmis par les lignes d'arbres. Pour analyser en détail les caractéristiques essentielles du comportement dynamique des transmissions, nous proposons une modélisation dynamique globale intégrant l'engrenage, les lignes d'arbres, les roulements et le carter. Nous calculons ensuite la réponse vibratoire de la transmission à l'aide d'une méthode originale, puis, nous analysons, sur un exemple, l'influence des propriétés mécaniques de chaque composante sur les mécanismes de transfert entre l'excitation générée par l'engrènement et la réponse vibratoire du carter. 


\section{Calcul de la réponse vibratoire de la transmission étudiée}

\subsection{Caractéristiques de la transmission}

Pour la présente étude, nous avons choisi une transmission qui possède toutes les caractéristiques essentielles des transmissions existantes. Il s'agit d'une boîte d'inversion de vitesse équipée d'un engrenage parallèle à denture hélicoïdale 49/49 dents.

\begin{tabular}{|c|c|c|}
\hline $\mathrm{Z}$ & 49 & 49 \\
\hline Rayon de base $\mathrm{R}_{\mathrm{b}}(\mathrm{mm})$ & 80.58 & 80.58 \\
\hline Angle de pression $\alpha$ & \multicolumn{2}{|c|}{$20^{\circ}$} \\
\hline Angle d'hélice $\beta$ & \multicolumn{2}{|c|}{$20^{\circ}$} \\
\hline Module $\mathrm{m}_{\mathrm{n}}(\mathrm{mm})$ & \multicolumn{2}{|c|}{3.5} \\
\hline Largeur b $(\mathrm{mm})$ & 35 \\
\hline Entraxe a' (mm) & \multicolumn{2}{|c|}{171.5} \\
\hline Rapport de conduite $\varepsilon_{\gamma}$ & 2.878 \\
\hline
\end{tabular}

Tableau 1. Caractéristiques de l'engrenage.

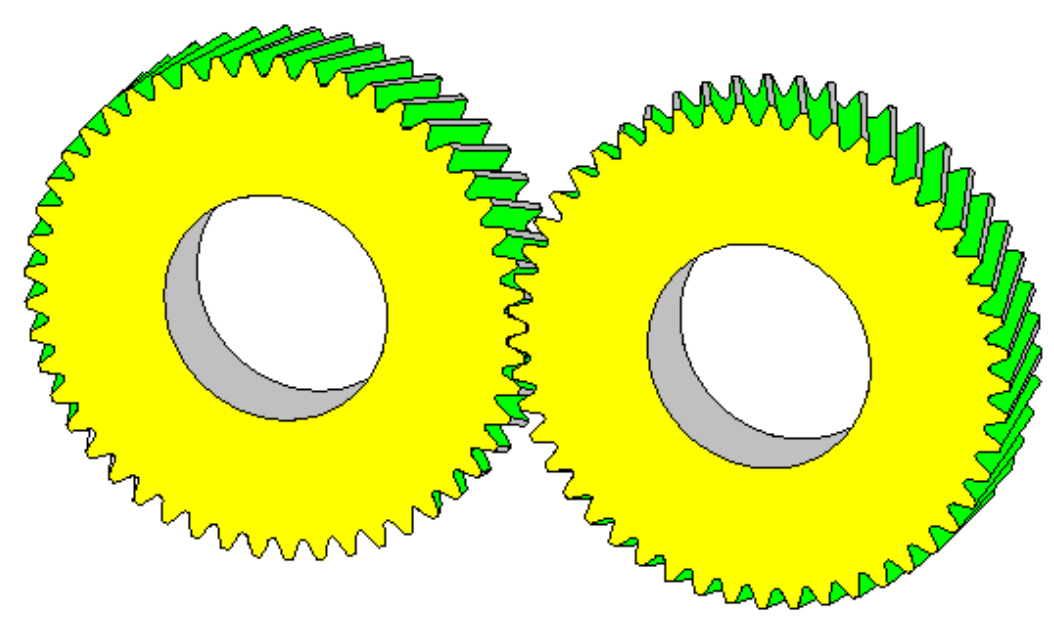

Figure 1. Caractéristiques de l'engrenage.

Les lignes d'arbres sont supportées par des roulements à rouleaux coniques. L'ensemble de ces composantes est intégré dans un carter en acier (450x280x160 mm) d'épaisseur $10 \mathrm{~mm}$ et en forme de parallélépipède rectangle. 


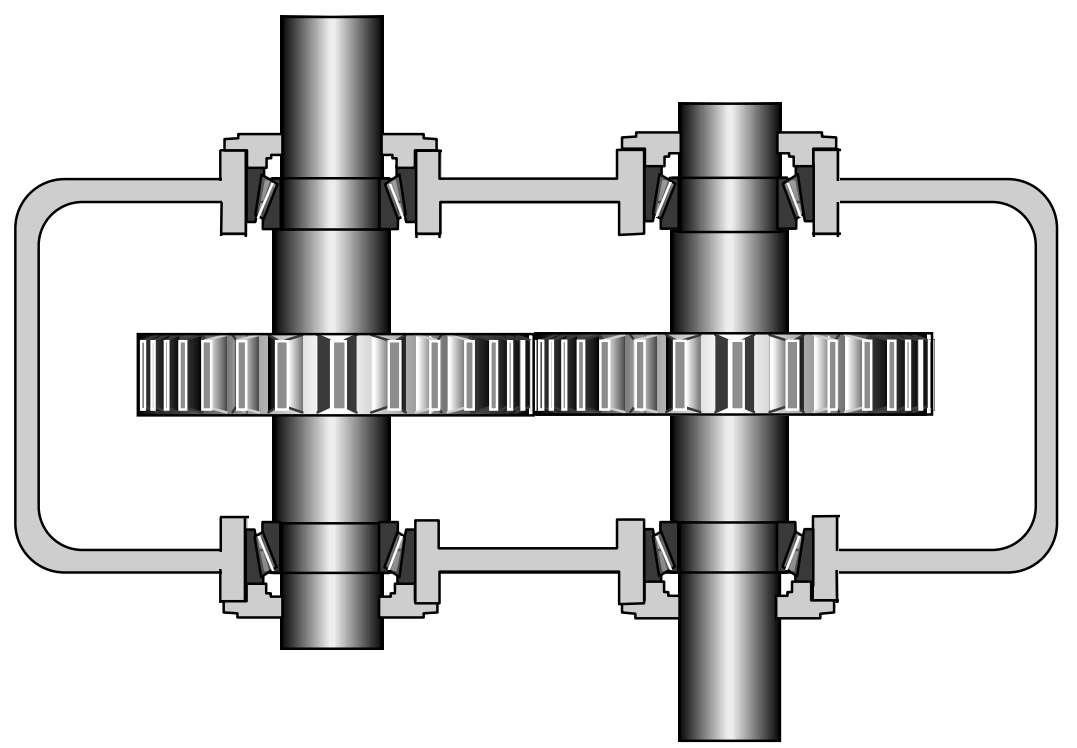

Figure 2. Transmission étudiée.

\subsection{Modélisation de la transmission par éléments finis}

La transmission est modélisée par la méthode des éléments finis. Les arbres sont discrétisés par des éléments de poutre à 2 nœuds et 6 degrés de liberté par nœud. Chaque roue dentée est modélisée par des éléments de masse et d'inertie concentrée rapportés sur les lignes d'arbres. L'engrènement est modélisé par un élément spécifique. Il s'agit d'une matrice de raideur généralisée de rang 12 qui couple les 6 degrés de liberté de la roue menée aux 6 degrés de liberté de la roue menante [PER 92, RIG 98]. Cette matrice est définie à partir des caractéristiques géométriques de l'engrenage et de la valeur moyenne de la raideur d'engrènement $\left(\mathrm{k}_{\mathrm{e}}=4.10^{8} \mathrm{~N} / \mathrm{m}\right)$ estimée lors du calcul de l'erreur statique de transmission [RIG 99]. Dans le repère local lié à l'engrenage, elle prend la forme suivante :$$
\underline{\underline{K}}=k_{\mathrm{e}} \cdot \underline{T}^{\mathrm{t}} \cdot \mathbf{T}
$$$$
\text { avec } I=\left(\quad 0, \quad \mathbf{1}, \quad \tan \beta, \quad \mathbf{R}_{b 1} \cdot \tan \alpha \cdot \tan \beta, \quad-\mathbf{R}_{b 1} \cdot \tan \beta, \quad \mathbf{R}_{b 1},\right.
$$$$
\left.0, \quad-1, \quad-\tan \beta, \quad R_{b 2} \cdot \tan \alpha \cdot \tan \beta, \quad-R_{b 2} \cdot \tan \beta, \quad R_{b 2}\right)
$$

Les inerties correspondant au moteur et à la charge sont reliées aux arbres via des raideurs en torsion modélisant les accouplements flexibles.

Les roulements induisent un couplage élastique entre les arbres et le carter selon trois directions orthogonales et deux angles de rotation. Leurs raideurs sont des fonctions non-linéaires de la charge transmise, en raison de la nature hertzienne des contacts étroits entre éléments roulants et bagues. Nous modélisons donc chaque 
roulement par une matrice de raideur généralisée de rang 10, en linéarisant le comportement du roulement autour de la position d'équilibre statique [LIM 90] (les raideurs axiales, radiales et angulaires sont égales à $\mathrm{K}_{\mathrm{z}}=10^{8} \mathrm{~N} / \mathrm{m}, \mathrm{K}_{\mathrm{x}}=\mathrm{K}_{\mathrm{y}}=10^{9} \mathrm{~N} / \mathrm{m}$ et $\left.\mathrm{K}_{\theta \mathrm{x}}=\mathrm{K}_{\theta \mathrm{y}}=10^{6} \mathrm{Nm} / \mathrm{rad}\right)$.

Le carter est discrétisé à l'aide d'éléments de plaques à 4 nœuds et 6 degrés de liberté par nœud et d'éléments volumiques à 8 nœuds et 3 degrés de liberté par nœuds qui représentent les supports des boîtiers de roulements.

Le modèle de la boîte d'inversion de vitesse complète possède environ 11000 degrés de liberté.

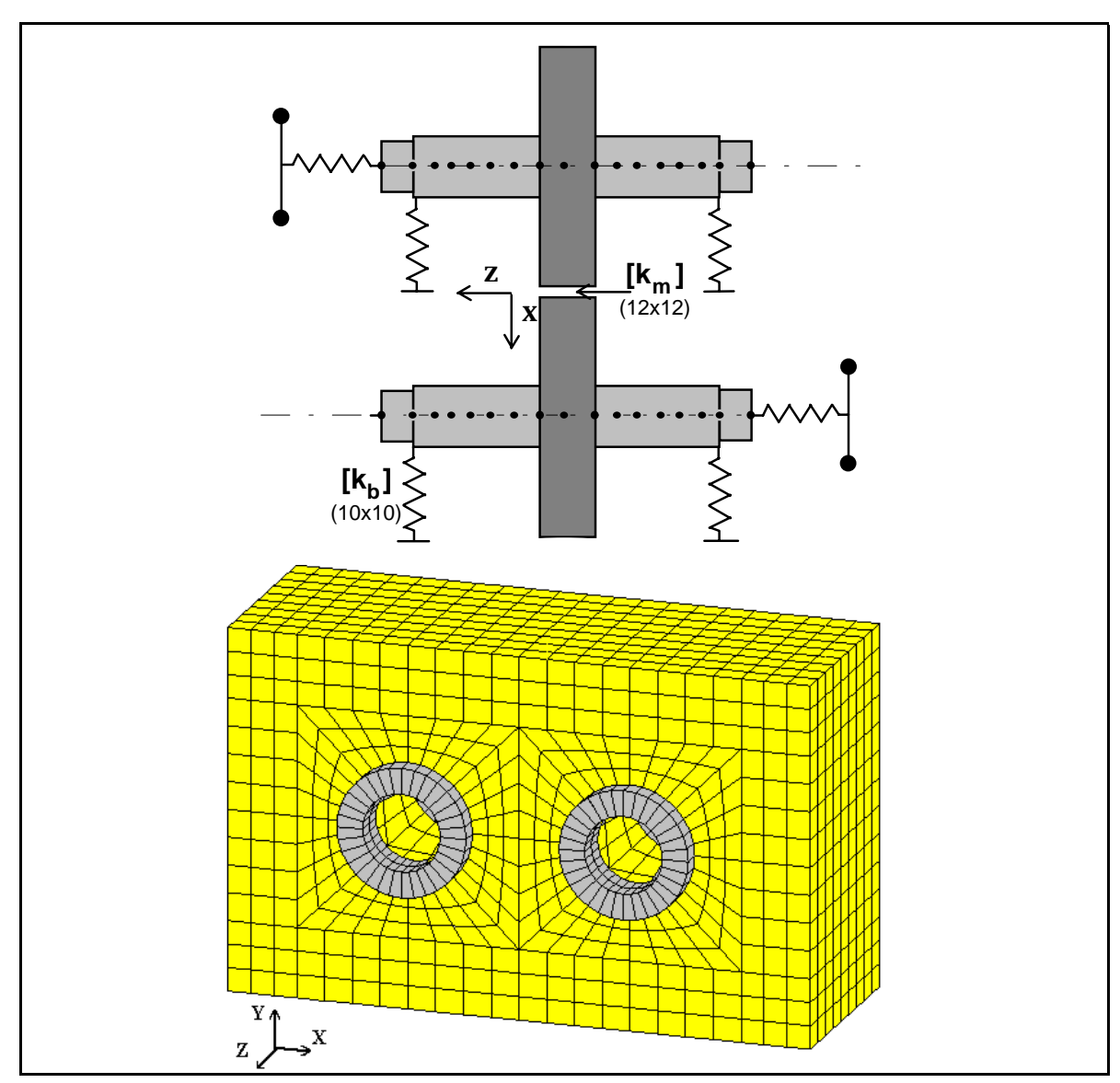

Figure 3. Modélisation des lignes d'arbres et du carter. 


\subsection{Analyse modale de la transmission}

Nous effectuons une analyse modale de la transmission par une méthode de condensation de Guyan, à partir des caractéristiques de raideur moyenne du système. Pour cela, nous définissons 600 degrés de liberté maîtres. Parmi ceux-ci, nous en sélectionnons 12 qui correspondant aux degrés de liberté de l'engrenage et 40 qui correspondent à ceux des roulements. Les autres sont équi-répartis pour permettre une bonne description des vibrations des arbres et du carter.

L'interprétation du comportement dynamique de la boîte d'inversion de vitesse nécessite l'analyse de ses formes propres et l'identification de certains modes particuliers que nous appelons "modes de denture" et "modes de roulement". Nous définissons ces modes à partir d'une approche énergétique [RIG 98]. Pour chacun des modes propres de la transmission, nous définissons $\mathbf{U}_{\mathbf{e}}$ l'énergie de déformation associée à la raideur d'engrènement et $\mathbf{U}_{\mathbf{T}}$ l'énergie totale de déformation :

Pour le j-ième mode,

$$
\begin{aligned}
& U_{\mathrm{e}}^{(j)}=\frac{1}{2}{ }^{\mathrm{t}} \underline{\Phi}^{(j)} \cdot \underline{\underline{k}}_{e} \cdot \underline{\Phi}^{(j)} \\
& U_{T}^{(j)}=\frac{1}{2}{ }^{\mathrm{t}} \underline{\Phi}^{(j)} \cdot \underline{\underline{\mathrm{K}}}_{T} \cdot \underline{\Phi}^{(j)}
\end{aligned}
$$

où $\quad-\underline{\Phi}^{(j)}$ est le vecteur propre du $\mathrm{j}$-ième mode,

- $\underline{\underline{K}}_{\mathrm{T}}$ est la matrice de raideur de la transmission complète,

- $\underline{\underline{k}}_{\mathrm{e}}$ est la matrice de raideur généralisée associée à la raideur d'engrènement. A l'exception des termes situés sur les 12 lignes et les 12 colonnes associées aux degrés de liberté de l'engrenage, les termes de cette matrice, de dimension égale à $\underline{\underline{\mathrm{K}}}_{\mathrm{T}}$, sont nuls.

Nous définissons le taux d'énergie de déformation $\rho_{\mathrm{e}}$ associée à la raideur d'engrènement :

$$
\rho_{\mathrm{e}}^{(\mathrm{j})}=\frac{\mathbf{U}_{\mathrm{e}}^{(\mathbf{j})}}{\mathbf{U}_{\mathrm{T}}^{(\mathbf{j})}}
$$

Nous pouvons comparer les valeurs de $\rho_{\mathrm{e}}$ associées à chaque mode propre de la transmission et nous choisissons d'appeler "modes de denture" les modes qui présentent le taux d'énergie $\rho_{\mathbf{e}}$ le plus élevé.

La définition des modes de denture est donc basée sur deux comparaisons successives: (1) la comparaison, pour chaque mode propre, des énergies de déformation associées à chaque élément de la transmission et (2) la comparaison des énergies de denture associées à chaque mode.

De la même manière que nous avons défini les modes de denture, nous pouvons définir les modes de roulement. Pour chaque mode propre de la transmission, l'énergie de déformation associée au roulement est égale à : 


$$
\mathbf{U}_{\mathrm{r}}^{(j)}=\frac{1}{2}{ }^{\mathrm{t}} \underline{\Phi}^{(\mathrm{j})} \cdot \underline{\underline{k}}_{\mathrm{r}} \cdot \underline{\Phi}^{(\mathrm{j})}
$$

où $\underline{\underline{k}}_{r}$ est la matrice de raideur du roulement. A l'exception des termes situés sur les 10 lignes et les 10 colonnes associées aux degrés de liberté du roulement, les termes de cette matrice sont nuls. Nous définissons le taux d'énergie de déformation $\rho_{r}$ associée au roulement :

$$
\rho_{r}^{(j)}=\frac{\mathbf{U}_{r}^{(j)}}{U_{T}^{(j)}}
$$

Nous pouvons comparer les valeurs de $\rho_{r}$ associées à chaque mode propre de la transmission et nous choisissons d'appeler "modes de roulement" les modes qui présentent le taux d'énergie $\rho_{r}$ le plus élevé.

\subsection{Résolution des équations de mouvement}

La seule source d'excitation retenue est l'erreur statique de transmission sous charge (ces caractéristiques sont calculées selon une méthode décrite dans [RIG 99]).

Si nous supposons que le couple moteur est suffisant pour prévenir toute perte de contact entre dentures et que les efforts dynamiques restent suffisamment faibles devant les efforts statiques induits par le couple moteur, le comportement vibratoire de la transmission discrétisée par la méthode des éléments finis est décrit par un système d'équations différentielles linéaires à coefficients périodiques. Pour un régime stationnaire et par assemblage des différents éléments, ce système d'équations régissant la réponse forcée $\underline{X}(t)$ de la transmission peut s'écrire sous la forme matricielle suivante :

$$
\underline{\underline{M}} \underline{\ddot{X}}+\underline{\underline{C}} \underline{\dot{X}}+\underline{\underline{K}} \underline{\mathbf{X}}+\mathbf{k}(\mathbf{t}) \underline{\underline{D}} \underline{\underline{X}}=\underline{F}(\mathrm{t})
$$

Dans cette équation, $\underline{\underline{M}}$ et $\underline{\underline{K}}$ sont les matrices classiques de masse et de raideur fournies par la méthode des éléments finis. Le couplage élastique entre les roues en prise est introduit par le terme $\mathrm{k}(\mathrm{t})$ qui représente la variation de la raideur d'engrènement. La matrice $\underline{\underline{D}}$ est associée aux caractéristiques de l'engrenage et la matrice $\underline{\underline{\mathrm{C}}}$ représente les termes de dissipation que nous introduirons ultérieurement par des taux d'amortissement modaux visqueux équivalents. Enfin, le vecteur $\underline{F}(\mathrm{t})$ constitue les efforts généralisés associés aux excitations internes. En régime de fonctionnement stationnaire, la variation de la raideur d'engrènement est une fonction périodique du temps. Dans la base modale associée à l'équation matricielle homogène à coefficients constants, l'équation du mouvement de la transmission discrétisée s'écrit :

$$
\underline{\underline{m} \ddot{q}}+\underline{\mathbf{c} q}+\underline{\underline{\mathbf{k}}} \underline{\underline{q}}+\mathbf{g}(\mathbf{t}) \underline{\underline{\mathbf{d}}} \underline{\underline{q}}=\underline{\mathbf{s}}(\mathbf{t})
$$

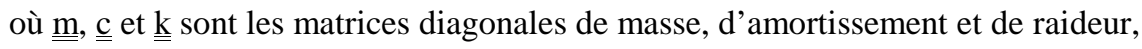
q est le vecteur des coordonnées modales, $\underline{s}(t)$ est le vecteur des forces généralisées, 
et $\underline{\underline{\mathrm{d}}}$ est la matrice non diagonale déduite des caractéristiques géométriques de l'engrenage et de la fluctuation centrée $\mathrm{g}(\mathrm{t})$ de la raideur périodique d'engrènement qui couple les équations de mouvement.

Lorsque l'excitation présente un spectre large bande, l'utilisation de méthodes de discrétisation temporelle peut conduire à des temps de calcul prohibitifs. La prise en compte des excitations à basse fréquence (fréquences de rotation des arbres) nécessite une discrétisation temporelle sur une période longue et la prise en compte des excitations à haute fréquence (fréquence d'engrènement et ses harmoniques) nécessite un pas de temps fin. Le nombre de pas nécessaire à la discrétisation temporelle des signaux devient donc très grand, ce qui nous a conduit à exploiter une autre méthode de résolution.

Perret-Liaudet [PER 94, PER 96] a développé une méthode baptisée méthode spectrale et itérative qui permet, en régime stationnaire, de résoudre les grands systèmes d'équations différentielles à coefficients périodiques en minimisant les temps de calcul. Cette méthode s'appuie sur une description spectrale de la fluctuation de la raideur d'engrènement et du vecteur d'excitation induit par les écarts de géométrie. La méthode utilisée fournit directement le spectre de la réponse vibratoire (amplitude et phase) pour chacun des degrés de liberté de la transmission.

\section{R ésultats}

\subsection{Surcharge dynamique de denture}

Lors de la conception d'une transmission par engrenages, il est important de prédire quelles sont les vitesses de rotation qui donnent naissance aux plus grandes surcharges dynamiques sur les dentures. Pour cela, nous avons calculé l'évolution de la valeur efficace de la surcharge dynamique de denture en fonction de la fréquence d'engrènement (figure 4). Nous avons introduit un coefficient d'amortissement visqueux équivalent égal à $3 \%$ pour chaque mode propre de la boîte d'inversion de vitesse.

Certains régimes de rotation donnent naissance à des amplifications de la surcharge dynamique de denture. Nous avons montré que ces «vitesses critiques» de rotation correspondent à l'excitation en résonance des modes de denture [RIG 96]. L'amplitude de la surcharge dynamique de denture est directement proportionnelle au taux d'énergie associée à la raideur d'engrènement.

Dans la littérature, ces vitesses critiques sont souvent prédites à partir de modélisation des lignes d'arbres en torsion pure ou en torsion-flexion. Nous avons montré que les déformations dynamiques des lignes d'arbres, des roulements et du carter ont une influence sensible sur ces modes de denture. Seule une modélisation globale intégrant l'ensemble des composantes de la transmission permet une prédiction correcte de ses vitesses critiques [RIG 96]. 


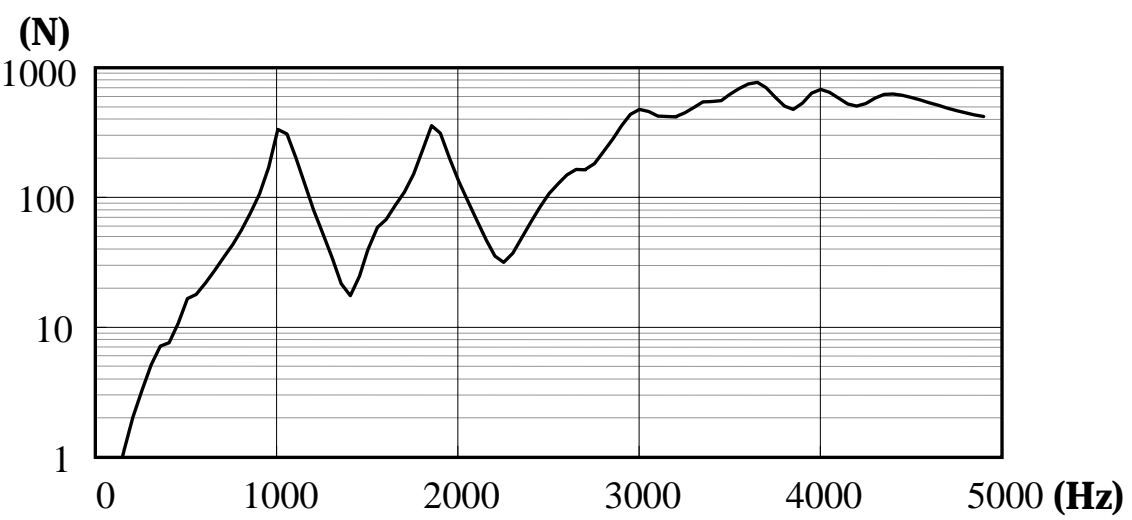

Figure 4. Evolution de la surcharge dynamique de denture avec la fréquence d'engrènement.

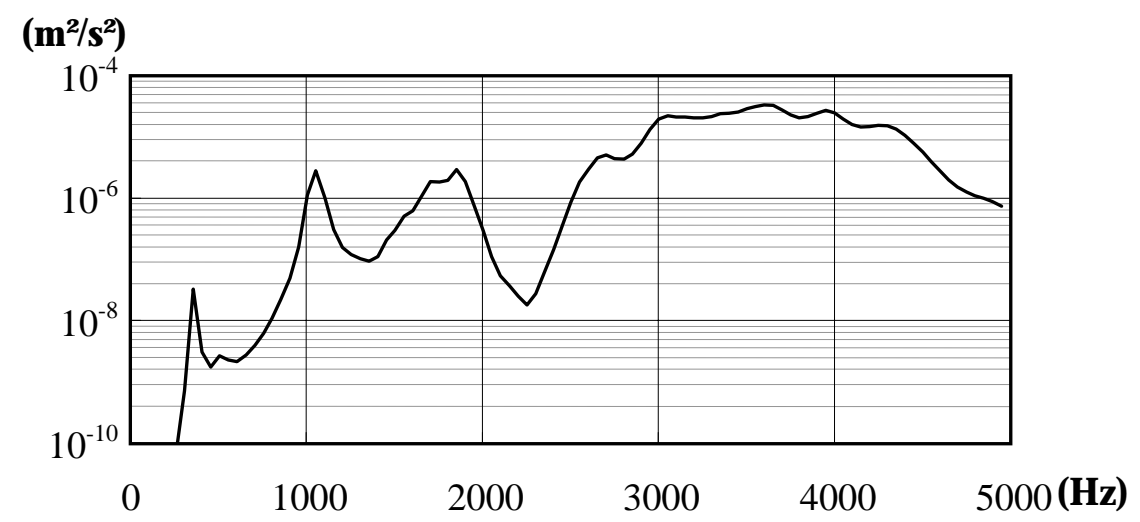

Figure 5. Evolution de la moyenne spatiale de la vitesse quadratique moyenne du carter avec la fréquence d'engrènement.

\subsection{Réponse vibratoire du carter}

Nous avons par ailleurs calculé l'évolution de la moyenne spatiale de la vitesse quadratique moyenne du carter définie comme suit :

$$
\left\langle\overline{V^{2}(\omega)}\right\rangle_{S}=\frac{1}{S} \int_{S}\left(\frac{1}{T} \int_{-T / 2}^{+T / 2} V^{2}(M, \omega, t) d t\right) d S
$$


Cette moyenne est proportionnelle, au facteur de rayonnement près, à la puissance acoustique rayonnée par la transmission.

La vitesse quadratique du carter présente des pics d'amplification entre 0 et $2500 \mathrm{~Hz}$ (figure 5) qui coïncident avec les vitesses critiques de rotation. D'autre part, elle atteint ses valeurs maximales entre 3000 et $4500 \mathrm{~Hz}$. Cette plage de fréquences correspond à celle où la surcharge dynamique de denture est, elle aussi, maximale. Ceci conduit à penser que les vitesses critiques de rotation sont en partie la cause des résonances de la vitesse quadratique du carter.

Pour comprendre les mécanismes de transfert entre la surcharge dynamique de denture et la vitesse quadratique du carter, nous avons calculé les efforts dynamiques transmis au carter par les roulements. Nous avons montré que leurs valeurs maximales correspondent à l'excitation des modes qui sont à la fois mode de denture et mode de roulement.

\begin{tabular}{|c|c|c|c|}
\hline Fréquence & $\boldsymbol{\rho}_{\mathrm{e}}$ & $\boldsymbol{\rho}_{\mathrm{y}}$ & $\sqrt{ }\left(\boldsymbol{\rho}_{\mathrm{e}} \boldsymbol{\rho}_{\mathrm{y}}\right)$ \\
\hline Mode 7 & $\mathbf{9 . 6} \%$ & $\mathbf{4 . 0} \%$ & $\mathbf{6 . 2} \%$ \\
\hline Mode 12 & $0 \%$ & $\mathbf{1 6 . 4} \%$ & $0 \%$ \\
\hline Mode 50 & $\mathbf{1 1 . 3} \%$ & $0 \%$ & $0.2 \%$ \\
\hline
\end{tabular}

Tableau 2. Taux d'énergie de déformation associés à la raideur d'engrènement et à la raideur radiale d'un des quatre roulements.

Le tableau 2, qui présente, pour trois modes particuliers, les taux d'énergie de déformation associés à la raideur d'engrènement et à la raideur radiale d'un des quatre roulements, permet d'illustrer ce résultat. Quatre situations sont possibles : - le mode est à la fois un mode de denture et un mode de roulement (mode 7), - le mode est un mode de roulement mais pas un mode de denture (mode 12), - le mode est un mode de denture mais pas un mode de roulement (mode 50), - le mode n'est ni un mode de denture, ni un mode de roulement.

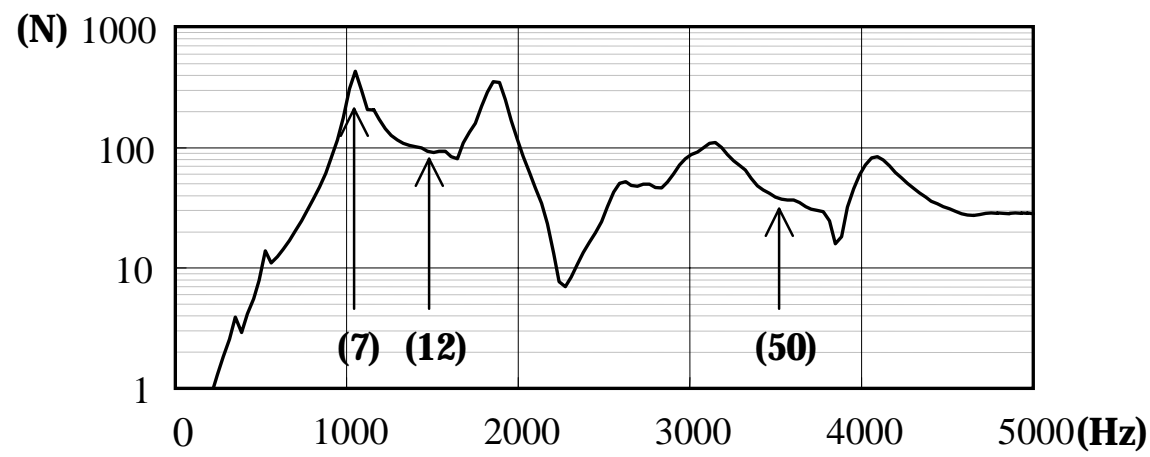

Figure 6. Effort radial $F_{y}$ transmis au carter par l'un des quatre roulements. 
Comme l'illustre la figure 6 , seule l'excitation du $7^{\text {ème }}$ mode donne naissance à une amplification de l'effort radial transmis au carter. L'amplitude de la surcharge est proportionnelle au facteur $\left(\rho_{\mathrm{e}} \cdot \rho_{\mathrm{y}}\right)^{1 / 2}$.

Les nombreuses simulations numériques que nous avons effectuées ont montré que ce résultat peut être généralisé à l'ensemble des efforts et des moments transmis au carter par les roulements. L'excitation d'un mode engendre une amplification des efforts dynamiques transmis au carter si et seulement si ce mode est à la fois un mode de denture et un mode de roulement.

Le calcul de l'ensemble des efforts internes généralisés transmis au carter à partir de la modélisation globale de la boîte d'inversion, de vitesse nous permet de valider le principe de réciprocité : pour chaque régime de fonctionnement, l'application simultanée de tous ces efforts sur le carter nu permet bien de retrouver la réponse vibratoire du carter calculée de manière. Par contre, l'excitation du carter nu par des efforts calculés avec un carter rigide conduirait à des résultats erronés car les propriétés mécaniques du carter modifient la nature de ces efforts [RIG 98].

Il est donc possible d'analyser les contributions respectives de chaque type d'effort à la réponse vibratoire du carter. Pour cela, nous avons calculé la vitesse quadratique du carter induite par les seuls efforts axiaux, puis par les seuls efforts radiaux, et enfin par les seuls moments dynamiques transmis au carter par les roulements. Les résultats obtenus nous conduisent aux conclusions suivantes :

- Les fréquences pour lesquelles la réponse vibratoire du carter est maximale correspondent à celles pour lesquelles les efforts et/ou les moments dynamiques transmis au carter par les roulements sont maximaux. Elles correspondent donc à l'excitation des modes qui sont à la fois mode de denture et mode de roulement.

- L'effet de chaque type d'effort (efforts axiaux, efforts radiaux ou moments) et, par conséquent, les solutions pour réduire la réponse vibroacoustique du carter dépendent de la fréquence considérée.

- Les modes pour lesquels l'énergie de déformation élastique est principalement associée au carter ne sont pas excités en résonance.

- La vitesse quadratique du carter de la boîte d'inversion de vitesse étudiée est induite essentiellement par les efforts axiaux entre 0 et $1000 \mathrm{~Hz}$, par les efforts radiaux entre 1000 et $2500 \mathrm{~Hz}$. Dans la plage de fréquence ou cette vitesse quadratique est maximale $(2500-5000 \mathrm{~Hz})$, elle est induite par les moments dynamiques transmis par les roulements.

\subsection{Influence des roulements et du carter}

Nous avons introduit des roulements à billes à contact obliques à la place des roulements à rouleaux coniques afin d'analyser l'influence des raideurs angulaires de ces roulements $\left(10^{5} \mathrm{Nm} / \mathrm{rad}\right.$ au lieu de $\left.10^{6} \mathrm{Nm} / \mathrm{rad}\right)$ sur la surcharge dynamique de denture et la vitesse quadratique du carter. 
(N)

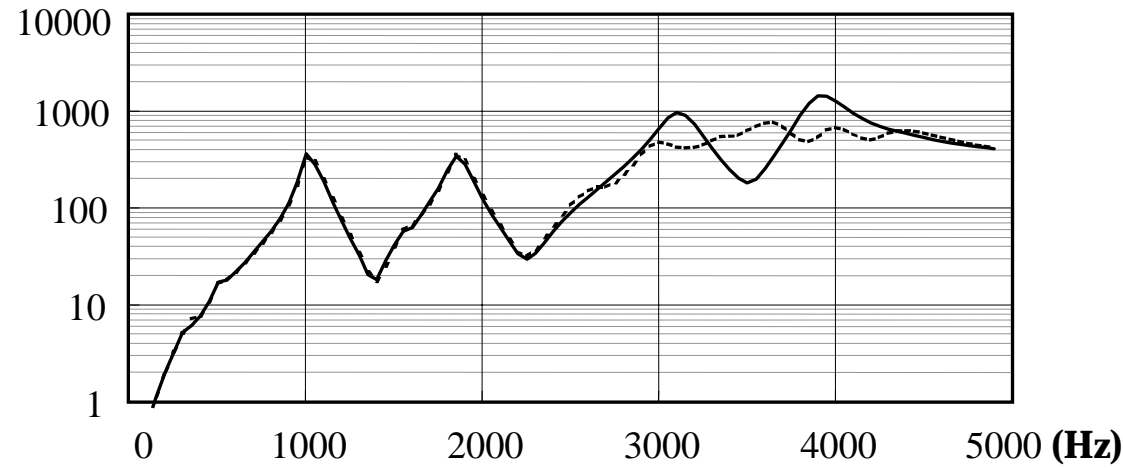

Figure 7. Evolution de la surcharge dynamique de denture.

Raideurs angulaires égales à $10^{5} \mathrm{Nm} / \mathrm{rad}(-)$ et $10^{6} \mathrm{Nm} / \mathrm{rad}(-----)$.

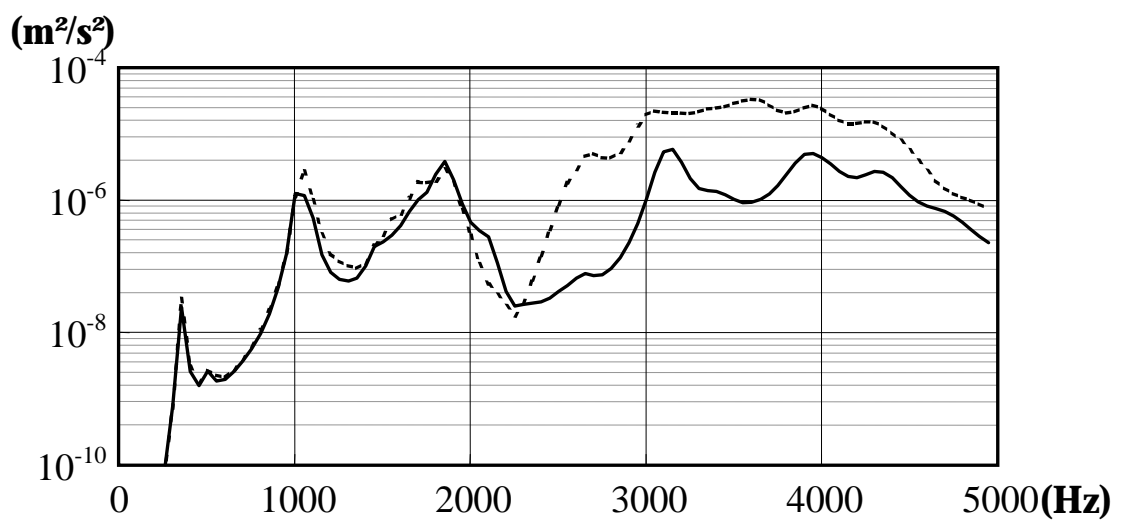

Figure 8. Evolution de la moyenne spatiale de la vitesse moyenne quadratique du carter. Raideurs angulaires égales à $10^{5} \mathrm{Nm} / \mathrm{rad}(-)$ et $10^{6} \mathrm{Nm} / \mathrm{rad}(-----)$.

Nous constatons tout d'abord que l'introduction des nouveaux roulements conduit à l'apparition de deux nouveaux modes de denture situés entre 2500 et $5000 \mathrm{~Hz}$. Comme le montre la figure 7 , l'excitation de ces modes génère des amplifications de la surcharge dynamique de denture.

Par ailleurs, les moments dynamiques transmis au carter par ces roulements sont beaucoup plus faibles que pour des raideurs angulaires de $10^{6} \mathrm{Nm} / \mathrm{rad}$, mais l'effet sur la vitesse quadratique du carter est différent selon la plage de fréquences 
considérée (figure 8). Elle dépend de la nature des efforts qui contribuent de façon majoritaire à la réponse vibratoire du carter. Ainsi, la vitesse quadratique du carter entre 0 et $2500 \mathrm{~Hz}$ a peu évolué car elle est induite en grande partie par les efforts radiaux et axiaux transmis par les roulements. Par contre, entre 2500 et $5000 \mathrm{~Hz}$, bien que la valeur maximale de la surcharge de denture ait augmenté, la diminution des raideurs angulaires des roulements conduit à une diminution sensible de la vitesse quadratique du carter.

Pour évaluer les effets dus à la modification des roulements, nous nous appuyons sur la comparaison des résultats obtenus pour chaque configuration de la boîte d'inversion de vitesse. Nous introduisons les indices de modification de la réponse vibratoire du carter $\mathbf{L}_{\mathbf{v}^{2}}$ et de la surcharge dynamique de denture $\mathbf{L}_{\mathbf{F}^{2}}$, définis entre 100 et $5000 \mathrm{~Hz}$, en bandes larges tiers d'octave.

$$
\begin{gathered}
L_{v^{2}}(\omega)=10 \cdot \log \left[\left\langle\overline{v^{2}(\omega)}\right\rangle /\left\langle\overline{v^{2}(\omega)}\right\rangle_{0}\right] \quad(\text { en dB) } \\
L_{F^{2}}(\omega)=10 \cdot \log \left[F^{2}(\omega) / F_{0^{2}}(\omega)\right] \text { en }(\mathrm{dB})
\end{gathered}
$$

où $\left\langle\mathbf{v}^{2}(\omega)\right\rangle$ et $\left\langle\mathbf{v}^{2}(\omega)\right\rangle_{0}$ sont les moyennes spatiales de la vitesse quadratique moyenne correspondant à la nouvelle configuration et à la solution de référence. $\mathbf{F}^{2}(\omega)$ et $\mathbf{F}_{0}{ }^{2}(\omega)$ sont les valeurs quadratiques des surcharges dynamiques de denture correspondant à la nouvelle configuration et à la configuration de référence.

L'analyse des indices en bandes larges tiers d'octave montre que la vitesse quadratique du carter est atténuée de -5 à $-16 \mathrm{~dB}$ bien que la surcharge dynamique de denture ait augmenté de $3 \mathrm{~dB}$ pour les régimes correspondant à l'excitation des nouveaux modes de denture (3200 et $4000 \mathrm{~Hz}$ ) (figure 9).

\section{(dB)}

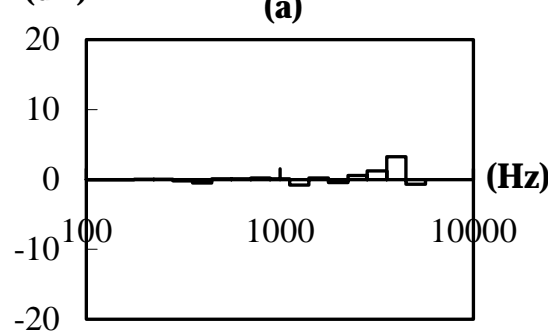

(dB)

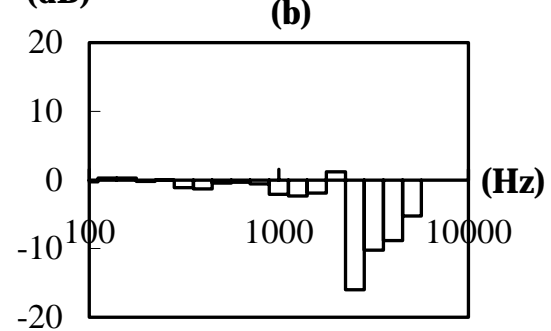

Figure 9. Indices de modification de la surcharge dynamique de denture (a) et de la vitesse quadratique du carter (b). Raideurs angulaires égales à $10^{5} \mathrm{Nm} / \mathrm{rad}(-)$ et $10^{6} \mathrm{Nm} / \mathrm{rad}(-----)$.

Une autre solution pour réduire la réponse vibratoire du carter d'une transmission consiste à modifier son architecture. Nous avons donc testé différentes configurations inspirées de solutions proposées par des auteurs qui ont analysé la 
réponse d'un carter nu soumis à un effort axial extérieur. Pour chaque configuration testée, nous avons adapté l'épaisseur du carter pour maintenir sa masse constante [RIG 98]. Parmi les solutions envisagées (augmentation de la masse des boîtiers de roulements, introduction d'un carter de forme ovale ...), seule l'introduction d'une nervure dans le plan contenant les roulements a eu un effet positif (figure 10). Elle se traduit par de fortes variations de l'indice $\mathrm{L}_{\mathrm{F}^{2}}$ qui confirment l'effet des propriétés mécaniques du carter sur les vitesses critiques (certaines vitesses critiques disparaissent tandis que de nouvelles vitesses critiques apparaissent). Elle induit surtout une atténuation sensible de la vitesse quadratique du carter dans une large bande de fréquences $(-10 \mathrm{~dB}$ entre 2500 et $5000 \mathrm{~Hz}$, figure 11). Cette atténuation s'ajoute à celle obtenue en modifiant les roulements.

Nous avons par ailleurs, constaté que seul un calcul couplé des réponses vibratoires des lignes d'arbres et du carter conduit à une analyse correcte des conséquences d'une modification de l'architecture d'une transmission par engrenages.

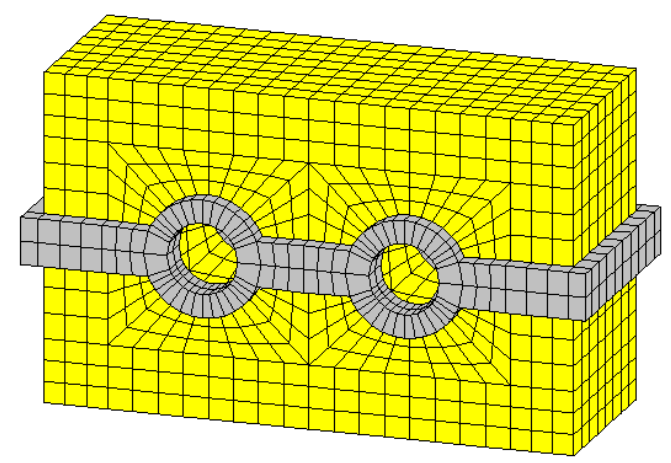

Figure 10. Modification du carter.
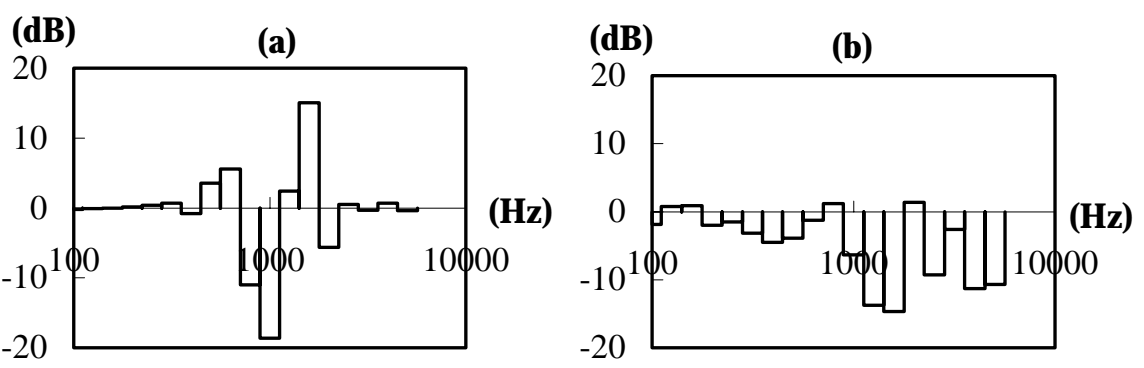

Figure 11. Indices de modification de la surcharge dynamique de denture (a) et de la vitesse quadratique du carter (b). Effet de la modification du carter. 


\section{Conclusion}

Nous avons choisi de nous intéresser au comportement vibratoire d'une boîte d'inversion de vitesse présentant toutes les caractéristiques essentielles des transmissions par engrenages. Pour calculer sa réponse vibratoire, nous avons adopté une approche globale basée sur une discrétisation par la méthode des éléments finis de l'ensemble des composantes de la transmission (engrenage, lignes d'arbres, roulements et carter). Nous avons résolu les équations de mouvement par une méthode spectrale et itérative. Nous nous sommes intéressés particulièrement à la surcharge dynamique de denture et à la vitesse quadratique du carter. Ce dernier paramètre est un indicateur de la puissance acoustique rayonnée par la boîte d'inversion de vitesse, même si certains modes peuvent être caractérisés par une plus grande efficacité de rayonnement acoustique que d'autres.

Les conclusions qui se dégagent des simulations numériques que nous avons effectuées sont les suivantes.

Parmi l'ensemble des modes propres d'une transmission par engrenages, certains présentent une énergie de déformation importante localisée au niveau de la raideur d'engrènement et/ou des roulements. Nous les avons appelés modes de denture et modes de roulement.

L'excitation en résonance des modes de denture est à l'origine d'une amplification de la surcharge dynamique de denture («vitesses critiques» de rotation). L'amplitude de la surcharge dynamique de denture est directement proportionnelle au taux d'énergie associée à la raideur d'engrènement. Seule une analyse globale qui intègre l'ensemble des composantes d'une transmission par engrenages peut permettre de prédire correctement ses vitesses critiques.

Les pics d'amplification de la moyenne spatiale de la vitesse quadratique moyenne du carter sont induits par des amplifications des efforts transmis au carter par les roulements. Ils correspondent à l'excitation en résonance de modes qui sont à la fois mode de denture et mode de roulements.

Seul un calcul couplé des réponses vibratoires des lignes d'arbres et du carter conduit à une analyse correcte des conséquences d'une modification de l'architecture d'une transmission par engrenages. Pour la boîte d'inversion de vitesse étudiée, nous avons montré que la diminution des raideurs angulaires des roulements et l'introduction d'une nervure dans le plan des roulements permettait d'atténuer sensiblement la vitesse quadratique du carter (tout en conservant sa masse constante).

La méthodologie adoptée constitue un outil qui permet d'améliorer les transmissions existantes ou de concevoir de nouvelles transmissions plus silencieuses. 


\section{Références}

[KAR 93] KaHRAman A. "Effect of Axial Vibrations on the Dynamics of a Helical Gear Pair ». Journal of Vibration and Acoustics, Vol. 115, p. 33-39, 1993.

[KAT 96] Kato M., Zhou H., Inoue K., Shibata K., Yasunami M. «Comments on gearbox housing structure for low noise ». VDI Berichte 1230, p. 765-777, 1996.

[LIM 90] LiM T., SingH R. « Vibration Transmission through Rolling Element Bearings. Part I : Bearing Stiffness Formulation. Part II : System Studies ». Journal of Sound and Vibration, Vol. 139 (2), p. 179-225, 1990.

[NER 88] NeriYa S., Bhat R., SANKAR T. «On the Dynamic Response of a Helical Geared System Subjected to a Static Transmission Error in the Form of a Deterministic and Filtered White Noise Input ». Journal of Vibration, Acoustics, Stress and Reliability in Design, Vol. 110, p. 501-506, 1988.

[OZG 88] ÖZgÜven H., Houser D. «Mathematical Models used in Gear Dynamics - A Review ». Journal of Sound and Vibration, Vol. 121 (3), p. 383-411, 1988.

[PER 92] PerRet-LiaudeT J. «Etude des mécanismes de transfert entre l'erreur de transmission et la réponse dynamique des boîtes de vitesses automobiles ». Thèse de doctorat de l'Ecole Centrale de Lyon N9207, 198 p., 1992.

[PER 94] PERRET-LIAUDET J. «Résolution de grands systèmes d'équations différentielles à coefficients périodiques: applications aux transmissions par engrenages ». Publications du LMA, premier colloque du GDR 1138 Vibroacoustique, Marseille, p. 55-68, 1994.

[PER 96] PERRET-Liaudet J. «An original method for computing the response of a parametrically excited forced system ». Journal of Sound and Vibration, Vol. 196, p. 165177,1996

[REM 92] Remond D., Velex P., Sabot J. «Comportement dynamique et acoustique des transmissions par engrenages - Synthèse bibliographique ». Publication du CETIM, 192 p., 1992.

[RIG 96] Rigaud E., SAвот J. «Effect of elasticity of shafts, bearings, casing and couplings on the critical rotational speeds of a gearbox ». VDI Berichte 1230, p. 833-845, 1996.

[RIG 98] Rigaud E. «Interactions dynamiques entre denture, lignes d'arbres, roulements et carter dans les transmissions par engrenages ». Thèse de l'Ecole Centrale de Lyon, 1998.

[RIG 99] Rigaud E., BARDAY D. «Modelling and Analysis of Static Transmission Error. Effect of Wheel Body Deformation and Interactions between Adjacent Loaded Teeth». 4th World Congress on Gearing and Power Transmission, Paris, Vol. 3, p. 1961-1972, 1999.

[TAK 91] Takatsu N., Kato M., Inoue K., Ishikawa M. « Analysis and experiment on the vibration transmission in a single stage gearbox ». International conference on motion and power transmissions, p. 104-109, 1991.

[WEL 79] WeLbouRn D. «Fundamental knowledge of gear noise- A survey ». Conference on noise and vibrations of engines and transmissions, Cranfield, p. 9-29, 1979. 